T. Ohsawa

Nagoya Math. J.

Vol. 158 (2000), 95-98

\title{
NONEXISTENCE OF REAL ANALYTIC LEVI FLAT HYPERSURFACES IN $\mathbb{P}^{2}$
}

\author{
TAKEO OHSAWA
}

\begin{abstract}
A real hypersurface $M$ in a complex manifold $X$ is said to be Levi flat if it separates $X$ locally into two Stein pieces. It is proved that there exist no real analytic Levi flat hypersurfaces in $\mathbb{P}^{2}$.
\end{abstract}

1. Let $X$ be a complex manifold of dimension $n$. A closed subset $M \subset X$ is called a real hypersurface of class $C^{\alpha}$, for $0 \leq \alpha \leq \infty$ or $\alpha=\omega$, if $M$ is locally expressed, with respect to real analytic coordinates of $X$, as the graph of some function of class $C^{\alpha}$ in $2 n-1$ real variables.

2. If a real hypersurface $M \subset X$ divides $X$ locally into Stein domains, $M$ is said to be Levi flat. In case $M$ is of class $C^{2}$, this condition is equivalent to that the complex Hessian of a defining function of $M$ is identically zero on the analytic tangent bundle $T_{M}^{1,0}:=\left(T_{X}^{1,0} \mid M\right) \cap\left(T_{M} \otimes \mathbb{C}\right)$ of $M$.

3. There is an open question whether or not there exists a Levi flat real hypersurface in $\mathbb{P}^{2}$. One of the motivations for asking this comes from the theory of Pfaff forms (cf. [C]).

4. The purpose of the present note is to prove the following.

TheOREM. There exist no real analytic Levi flat hypersurfaces in $\mathbb{P}^{2}$.

5. Proof will be done by contradiction.

6. Suppose that there existed such $M \subset \mathbb{P}^{2}$. We may assume that $M$ is connected.

7. The following observations have long been known.

(i) The holomorphic normal bundle $N_{M}^{1,0}:=\left(T_{\mathbb{P}^{2}}^{1,0} \mid M\right) / T_{M}^{1,0}$ admits a fiber metric whose curvature form is positive along $T_{M}^{1,0}$.

Received March 24,1999.

2000 Mathematics Subject Classification: 32V40. 
(ii) $\mathbb{P}^{2} \backslash M$ is a Stein manifold with two connected components.

8. (i) is true because $N_{M}^{1,0}$ is, as a $C R$ line bundle, a quotient of a Griffithspositive bundle say $T_{\mathbb{P}^{2}}^{1,0} \mid M$. (That $T_{\mathbb{P}^{2}}^{1,0}$ is Griffiths-positive is equivalent to saying that $\mathbb{P}^{2}$ admits a metric whose holomorphic bisectional curvature is positive. It is straightforward that the Fubini-Study metric has this property.)

9. (ii) is true in virtue of A. Takeuchi $[\mathrm{T}]$. (For a somewhat simplified proof of Takeuchi's theorem, see $[\mathrm{O}]$.) That $\mathbb{P}^{2} \backslash M$ has two components follows from the fact that $\mathbb{P}^{2}$ is simply connected. It follows in particular that $M$ is orientable.

10. As in $[\mathrm{O}-\mathrm{S}]$ we put

$$
N_{M}:=T_{M} \otimes \mathbb{C} /\left(T_{M}^{1,0}+\overline{T_{M}^{1,0}}\right)
$$

Since the projection $T_{\mathbb{P}^{2}} \otimes \mathbb{C} \rightarrow T_{\mathbb{P}^{2}}^{1,0}$ induces an isomorphism between $N_{M}$ and $N_{M}^{1,0}$, we shall not distinguish them.

11. Since $M$ is Levi flat and of class $C^{\omega}, N_{M}$ admits a system of real analytic local frames such that the transition functions between them are real valued.

12. Moreover, since $M$ is orientable, these transition functions can be chosen to be positive.

13. More explicitly, real analytic defining functions of the Levi flat hypersurface $M$ are of the form Re $f$ for holomorphic $f$. Since $M$ is orientable, open sets of $\mathbb{P}^{2}$, say $U_{i}(i=1,2, \ldots, m)$, can be chosen in such a way that $M \subset \bigcup_{i=1}^{m} U_{i}$ and that one has a holomorphic function $f_{i}$ on $U_{i}$ such that $\operatorname{Re} f_{i}$ is a defining function of $M \cap U_{i}$ and

$$
e_{i j}:=\left.\frac{d f_{i}}{d f_{j}}\right|_{M \cap U_{i} \cap U_{j}}\left(=\frac{d\left(\operatorname{Im} f_{i} \mid M \cap U_{i} \cap U_{j}\right)}{d\left(\operatorname{Im} f_{j} \mid M \cap U_{i} \cap U_{j}\right)}\right)>0 .
$$

14. This implies that, for any positive integer $k$, there exists a holomorphic line bundle, say $L_{k}$, over some neighbourhood of $M$ in $\mathbb{P}^{2}$ satisfying

$$
L_{k}^{\otimes k} \mid M=N_{M}
$$


15. We shall prove that, for each $k$, at least one of such $L_{k}$ can be extended to a holomorphic line bundle over $\mathbb{P}^{2}$.

16. For that, because of the Steinness of $\mathbb{P}^{2} \backslash M$ and because $\operatorname{dim}\left(\mathbb{P}^{2} \backslash M\right)>$ 1 , it suffices to show that some $L_{k}$ is an analytic subsheaf of a coherently extendable locally free sheaf.

17. We note that this criterion of extendability is a corollary of a more general extension theorem due to S. Ivashkovitch [I], which asserts that, given any connected Stein manifold $S$ of dimension at least 2 and any compact Kähler manifold $Y$, holomorphic maps from the complement of any compact subset of $S$ to $Y$ extend meromorphically to $S$.

18. By this criterion, the extendability of $L_{1}$ is immediate because $L_{1}^{*} \subset$ $\left(T_{\mathbb{P}}^{1,0}\right)^{*}$. Hence the sheaf of the germs of holomorphic sections of $L_{1}$, denoted by $\mathcal{O}\left(L_{1}\right)$, is the restriction of some invertible sheaf $\mathcal{L} \rightarrow \mathbb{P}^{2}$.

19. By (i), the degree of $\mathcal{L}$ must be positive. This means that there exist two sections $s_{1}, s_{2} \in \Gamma\left(\mathbb{P}^{2}, \mathcal{L}\right)$ such that $s_{1}^{-1}(0) \cup s_{2}^{-1}(0)$ consists of $2 d$ complex lines of general position, where $d=\operatorname{deg} \mathcal{L}$, and that

$$
\operatorname{Sing}\left(s_{1}^{-1}(0) \cup s_{2}^{-1}(0)\right) \cap M=\emptyset .
$$

20. Let $\pi_{k}: X_{k} \rightarrow \mathbb{P}^{2}$ be the $k$-sheeted ramified covering that makes the $k$-th root of $s_{2} / s_{1}$ univalent outside the set of indeterminancy.

21. Then one can find a holomorphic section $\tau_{k} \in \Gamma\left(\pi_{k}^{-1}(U), \mathcal{O}\left(\pi_{k}^{*} L_{k}\right)\right)$ for a sufficiently small neighbourhood $U$ of $M$, such that $\tau_{k}^{k}=\pi_{k}^{*} s_{1}$, for some $L_{k}$.

22. Hence there exists $L_{k}$ such that $\pi_{k}^{*} L_{k}$ is the line bundle associated to the divisor $\frac{1}{k} \pi_{k}^{-1}\left(s_{1}^{-1}(0)\right)$.

23. Since $\pi_{k}^{*} L_{k}$ is extendable to $X_{k}, \pi_{k^{*}} \pi_{k}^{*} L_{k}$ is extendable to $\mathbb{P}^{2}$. But $L_{k}$ is a subbundle of $\pi_{k^{*}} \pi_{k}^{*} L_{k}$, so that $L_{k}$ is extendable, too.

24. Therefore $\operatorname{deg} \mathcal{L}$ is a positive integer which is divisible by any positive integer $k$, which is absurdity.

25. The following is an immediate consequence of the theorem.

COROLlary 1. If $\mathbb{P}^{n}$ admits a Levi flat real analytic hypersurface, $n \leq 1$. 


\section{REFERENCES}

[C] D. Cerveau, Minimaux des feuilletages algébriques de $\mathbb{C P}(n)$, Ann. Inst. Fourier, 43 (1993), 1535-1543.

[I] S. Ivashkovitch, The Hartogs-type extension theorem for meromorphic maps into compact Kähler manifolds, Invent. math., 109 (1992), 47-54.

[O] T. Ohsawa, Pseudoconvex domains in $\mathbb{P}^{n}$ : a question on the 1-convex boundary points, to appear in the proceedings of Taniguchi sypm.

[O-S] T. Ohsawa, and N. Sibony, Kähler identity on Levi flat manifolds and application to the embedding, Nagoya Math. J., 158 (2000), 87-93.

[T] A. Takeuchi, Domains pseudoconvexes infinits et la metrique riemannienne dans us espace projectif, J. Math. Soc. Japan, 16 (1964), 159-181.

Graduate School of Mathematics

Nagoya University

Chikusa-ku, Nagoya 464-8602

Japan 